\title{
Rapid Eye Movement Sleep Behavior Disorder in a Patient with Brainstem Lymphoma
}

\author{
Chen Jianhua ${ }^{1}$, Liu Xiuqin ${ }^{1}$, Cui Quancai ${ }^{2}$, Sun Heyang ${ }^{1}$ and Huang Yan ${ }^{1}$
}

\begin{abstract}
Rapid eye movement (REM) sleep behavior disorder (RBD) is common in patients with neurodegenerative diseases. RBD occurring in a patient with brainstem lymphoma has not yet been reported. A 30-year-old man had an eleven-month history of violent motor and vocal behavior during sleep. Brain magnetic resonance imaging disclosed hypointensity $\mathrm{T} 1$ and hyperintensity $\mathrm{T} 2$ signals in the pontomesencephalic junction and at the upper/mid pons level. A stereotactic biopsy demonstrated the presence of diffuse large B-cell lymphoma. On polysomnography, there was enhanced submental and limb electromyographic tone and increased muscular activity during REM sleep. Chemotherapy diminished the dream-enacting behaviors. This case provides evidence that treatment aimed at the primary disease can partially improve the frequency of RBD.
\end{abstract}

Key words: lymphoma, brainstem, REM sleep behavior disorder

(Intern Med 52: 617-621, 2013)

(DOI: 10.2169/internalmedicine.52.8786)

\section{Introduction}

Rapid eye movement (REM) sleep behavior disorder (RBD) is a parasomnia characterized by vigorous dreamenacting behaviors associated with nightmares and abnormally increased electromyographic activity during REM sleep $(1,2)$. It is thought that excessive electromyographic (EMG) activity during REM sleep in RBD patients reflects dysfunction of the brainstem structures responsible for muscle atonia during REM sleep (2-5). RBD is very common in several neurodegenerative diseases, including multiple system atrophy, dementia with Lewy bodies and Parkinson's disease (6-8). It is also rarely observed in some patients with focal lesions of the brainstem from various causes, including vascular diseases, multiple sclerosis and inflammatory lesions (9-18). We herein report the case of an RBD patient with brainstem lymphoma.

\section{Case Report}

A 30-year-old man presented with an eleven-month history of violent motor and vocal behavior during sleep. He would often suddenly awaken from fearful and vivid dreams with terrified screams or violent behavior such as hurling, gesturing or sitting up. Sometimes, he punched or kicked his spouse. The nocturnal behaviors mostly matched his dream content and occurred approximately one to two times per week.

The patient reported experiencing headaches, ptosis and diplopia one month before developing his sleep disorder, although he did not pay much attention to them at first. He had no history of depression, head injury, cigarette smoking or alcohol abuse. He had an unremarkable family and a personal history free from any medications. A neurological examination revealed right third nerve palsy and bilateral Babinski signs.

On brain magnetic resonance imaging (MRI), there were gadolinium-enhanced hypointense signals on T1-weighted images and hyperintense signals on T2-weighted images along with fluid-attenuated inversion recovery (FLAIR) sequences in the pontomesencephalic junction and nongadolinium-enhanced hypointensity $\mathrm{T} 1$ and hyperintensity T2 and FLAIR signals at the upper/mid pons level (Fig. 1). The cerebrospinal fluid contained 20 white blood cells/mcL, a protein level of $0.78 \mathrm{~g} / \mathrm{L}(0.15-0.45)$, a glucose level of

${ }^{1}$ Department of Neurology, Peking Union Medical College Hospital, Chinese Academy of Medical Sciences, China and ${ }^{2}$ Department of Pathology, Peking Union Medical College Hospital, Chinese Academy of Medical Sciences, China

Received for publication August 13, 2012; Accepted for publication September 25, 2012

Correspondence to Dr. Liu Xiuqin, liuxq6@yahoo.cn 


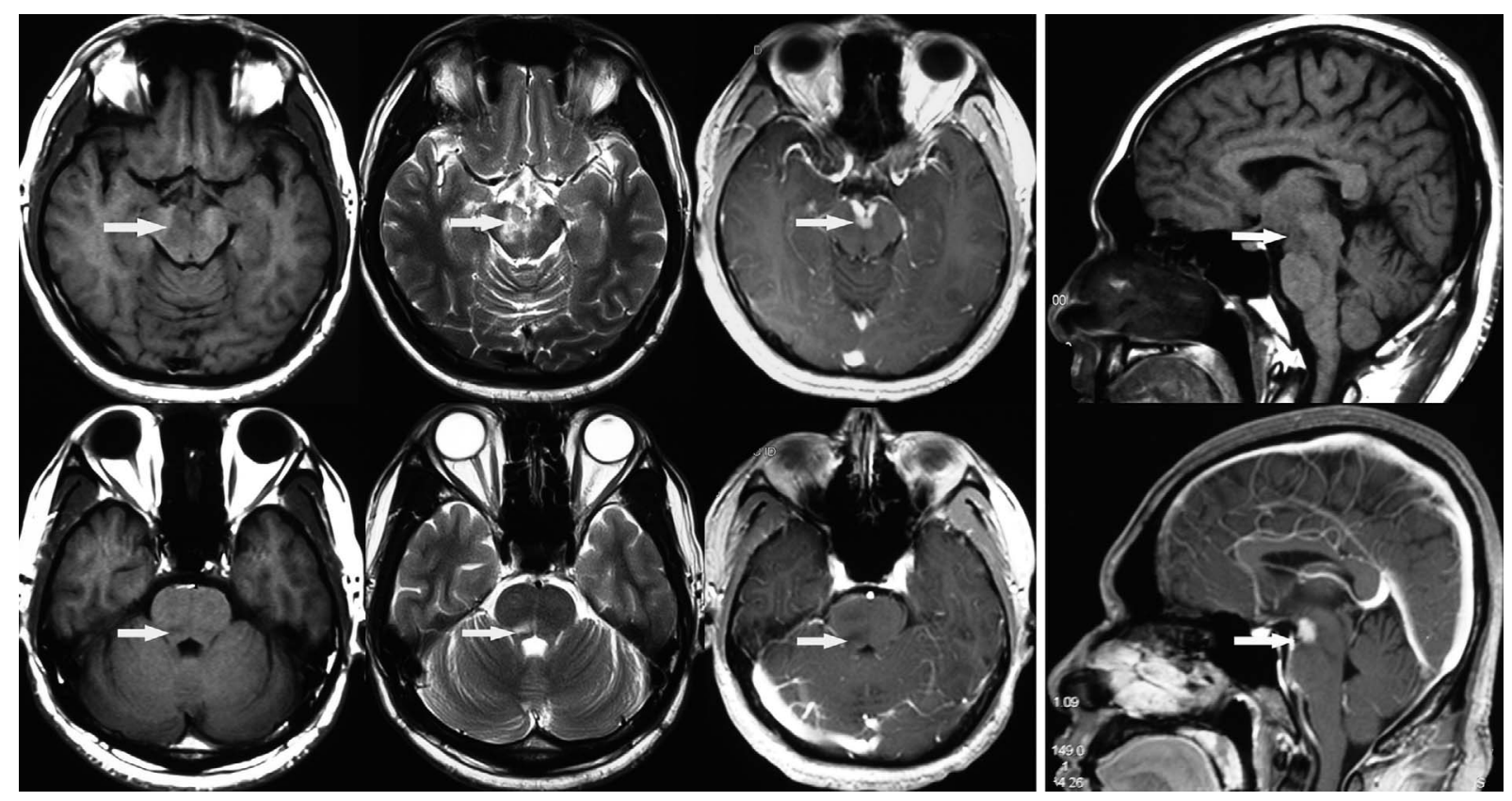

Figure 1. Axial and sagittal MRI images obtain at symptom onset showed gadolinium-enhanced hypointensity $\mathrm{T} 1$ and hyperintensity $\mathrm{T} 2$ signals in the pontomesencephalic junction and non-gadolinium-enhanced hypointensity $\mathrm{T} 1$ and hyperintensity $\mathrm{T} 2$ signals at the upper/mid pons level (arrow).

$2.5 \mathrm{mmol} / \mathrm{L}(2.5-4.4)$ and a chloride level of $116 \mathrm{mmol} / \mathrm{L}$ (118-128 mmol/L). Tests for autoimmune diseases, infections, sarcoidosis, vasculitis and cerebral vascular diseases were negative. Tests for paraneoplastic antineuronal antibodies and antibodies against Aquaporin 4 were negative. A presumptive diagnosis of demyelinating disease was made. Intravenous methylprednisolone was administered at a dose of $1,000 \mathrm{mg} /$ day for three days followed by prednisone tapered over one week. The patient's headache remitted without relief of ptosis and diplopia. His symptoms became aggravated eight months later. The bilateral visual evoked potentials, brainstem auditory evoked potentials, sensory evoked potentials and motor evoked potentials of the four limbs were normal. Brain MRI revealed enlargement of the brainstem lesions (Fig. 2). Computed tomography scans of the chest, abdomen and pelvis and the results of a bone marrow biopsy were normal. The patient was treated with corticosteroids and anti-inflammatory medications without showing any benefits.

A stereotactic brainstem biopsy was performed, which demonstrated diffuse large B-cell lymphoma (DLBCL). Hence, the patient was diagnosed with primary central nervous system lymphoma (PCNSL).

One year after the onset of symptoms, overnight polysomnography (PSG) was performed using an extended montage of EEG (F3/A2, F4/A1, C3/A2, C4/A1, Cz/A1, O1/A2, O2/ A1), EOG, oronasal airflow by thermistor, EMG of the chin and tibialis anterior muscles, respiratory inductance plethysmography, ECG, pulse oximetry and continuous behavioral monitoring with a video. The results of the PSG showed a total sleep time of 488.5 minutes, which consisted of
$23.83 \%$ stage 1 NREM sleep, $46.44 \%$ stage 2 NREM, $14.79 \%$ SWS, $14.94 \%$ stage REM and $12.03 \%$ stage REM sleep without atonia. REM latency and density was 163 minutes and $33.4 \%$, respectively. On the PSG, there was both enhanced phasic and tonic submental and limb EMG tone and increased muscular activity in the second and third periods of REM sleep (Fig. 3). The audiovideo recording showed the patient presenting with an episode of remarkable muscle twitching and jerking with violent kicking of his right leg and screaming. When awakened, he became well oriented and recalled very fierce fighting. No epileptiform activity or periodic limb movements (PLMS) were observed during sleep. No apneas or hypopneas were observed.

The patient received an IV infusion of methotrexate (MTX) at a dose of $2 \mathrm{~g} / \mathrm{m}^{2}$ over four hours on day 1 and cytarabine (AraC) at a dose of $3 \mathrm{~g} / \mathrm{m}^{2}$ every 12 hours on days 2 to 3 . He refused clonazepam treatment and was closely followed for six months. His condition stabilized. Repeat brain MRI performed after two courses of chemotherapy showed disappearance of the lesions. The occurrence of nocturnal motor and vocal behaviors diminished to approximately one to two times per month after the administration of two courses of chemotherapy. The severity of RBD improved, and the patient's night behaviors became less aggressive.

\section{Discussion}

PCNSL is an extranodal malignant lymphoma arising within the brain, eyes, leptomeninges or spinal cord in the absence of systemic lymphoma at the time of diagnosis. 


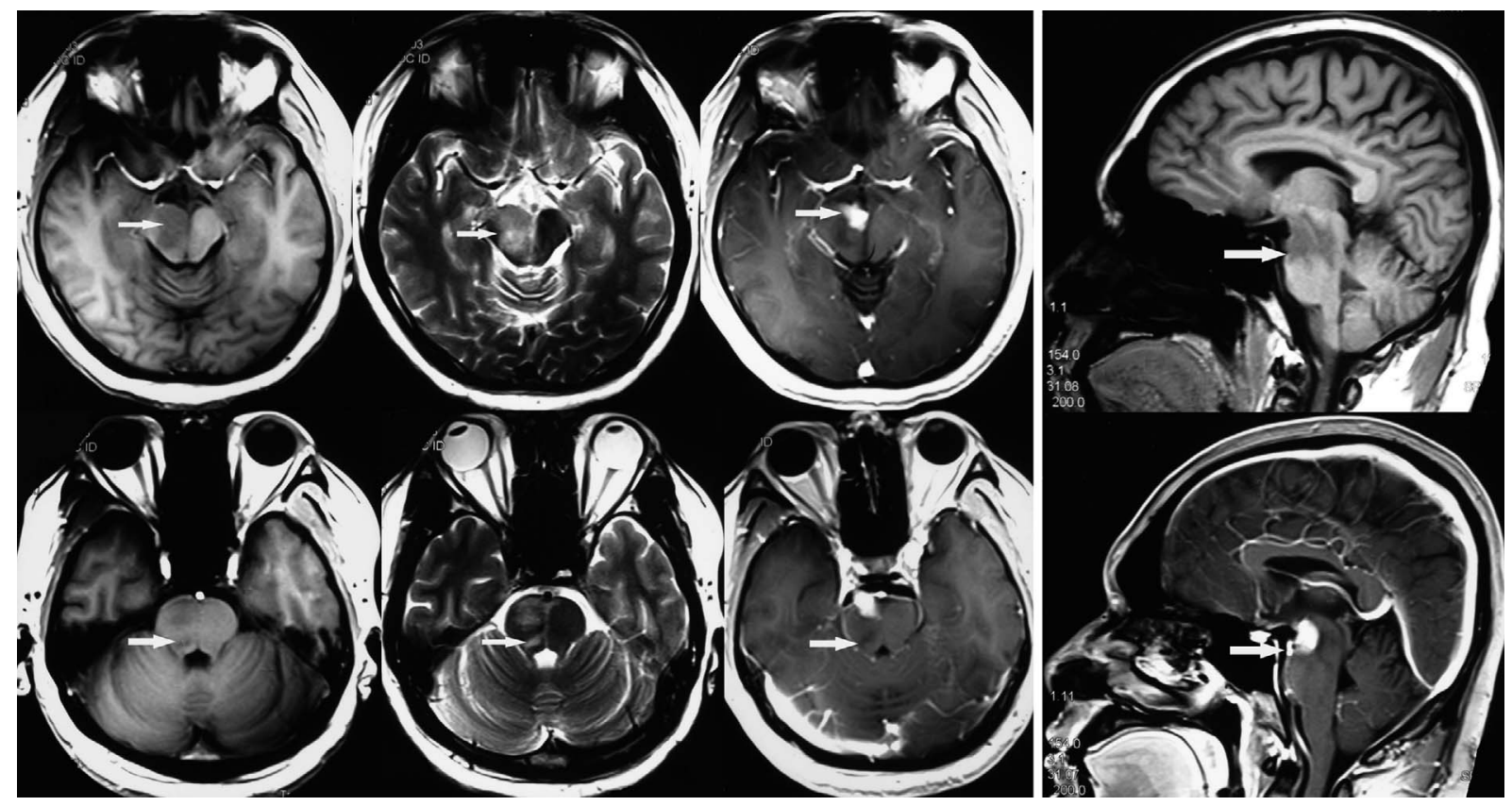

Figure 2. Axial and sagittal MRI images obtained one year after the onset of symptoms revealed that the lesions in the pontomesencephalic junction and upper/mid pons had become enlarged (arrow).

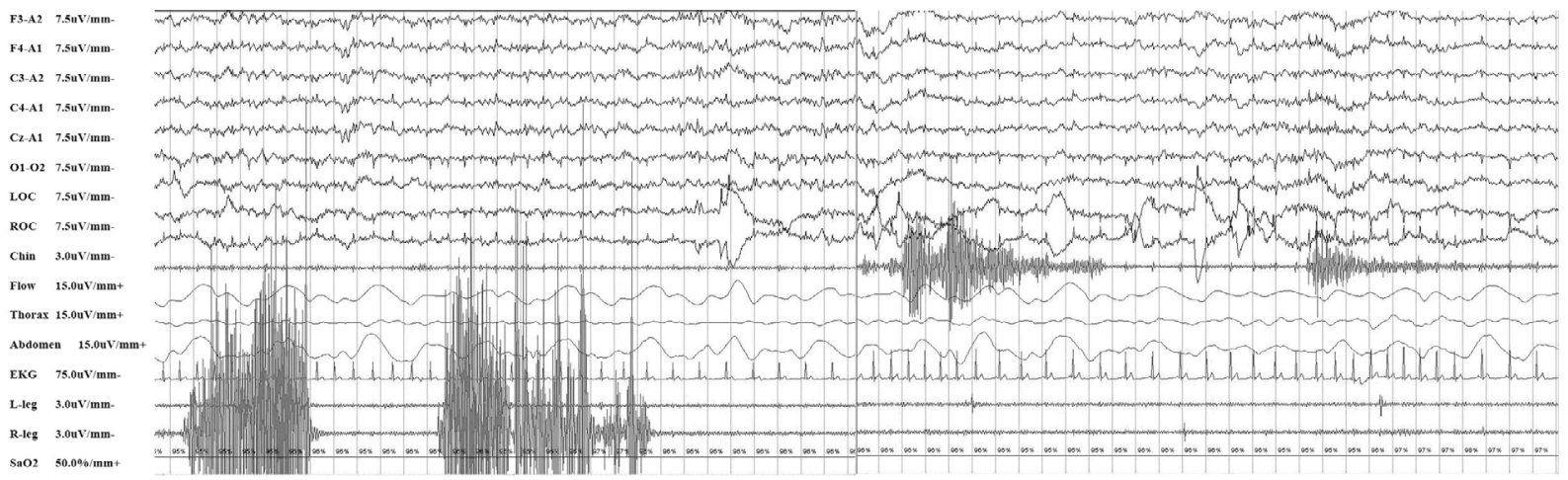

Figure 3. Thirty-second epoch polysomnograms showed REM sleep without atonia. There were bursts of increased activity without atonia in the tibial and chin EMG channels during REM sleep.

Lymphomas involving the central nervous system are increasingly recognized in immunocompetent as well as immunosuppressed individuals (19). In immunocompetent patients, only $5 \%$ of PCNSLs are DLBCLs (20). No systemic lymphoma evidence was found in this patient, which satisfied the diagnostic criteria for PCNSL with biopsy proof. The patient demonstrated violent motor and vocal behaviors during nocturnal sleep. On the PSG, abnormalities in muscle tone were observed during REM sleep without EEG epileptiform activity. These clinical features satisfied the diagnostic criteria for RBD (1). RBD onset in this case occurred one month after the onset of neurological symptoms of the brainstem lesions, and chemotherapy for the lymphoma was associated with decreased episodes of RBD. This suggests that brainstem lymphoma was the direct cause of RBD.

The brainstem regions classically considered to be involved in RBD pathophysiology based on lesion studies conducted in cats include the medullary magnocellular reticular formation (MMRF), the locus coeruleus/subcoeruleus complex, the pedunculopontine nucleus (PPN), the laterodorsal tegmental nucleus (LDT) and possibly the substantia nigra (SN) (21).

The critical structures in the brainstem involved in REM sleep control in humans include the "REM-off" region consisting of the ventrolateral part of the periaqueductal gray matter (vlPAG) and the lateral pontine tegmentum (LPT), the "REM-on" region consisting of the precoeruleus (PC) and the sublaterodorsal nucleus (SLD), as well as the extended part of the ventrolateral preoptic nucleus (eVLPO), locus coeruleus (LC), LDT, PPN and raphe nucleus (RN) $(21,22)$.

Interestingly, RBD has also been reported to occur in association with damage in the limbic system without primary brainstem impairment (23-25). Previous imaging studies 

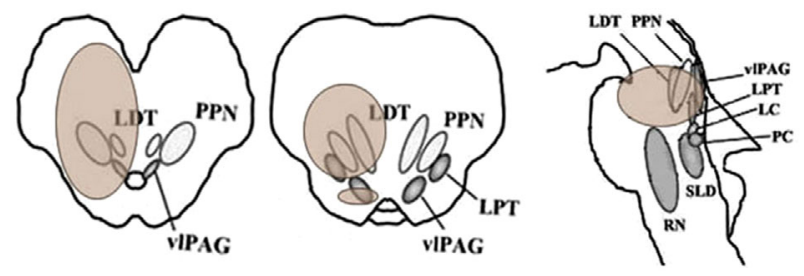

Figure 4. The approximate location of the lymphoma based on the MRI data shown on human brainstem templates. $₫$ The lesion in the patient. (This diagram was drawn in reference to data published by Boeve (21).)

have shown that, during REM sleep, regional cerebral blood flow is significantly increased in the pontine tegmentum, thalamic nuclei and limbic areas $(26,27)$. This interaction within the limbic system is activated during REM sleep, suggesting that limbic system impairment may be implicated in the pathogenesis of RBD (24).

Adams et al. reported a case of RBD associated with paraneoplastic neurologic disorder with multifocal involvement including the hypothalamus, hippocampus and brainstem despite the absence of lesions on MRI scans (28). This report hinted that RBD may be associated with autoimmune disorders involving the limbic system or brainstem.

We considered that the pathogenesis of the present case of RBD may be related to the lesions in the pontomesencephalic junction and the right tegmentum at the upper/mid pons level (Fig. 4). The PPN and LDT nuclei, which belong to the "REM-on" region, and the vlPAG and LPT nuclei, which belong to the "REM-off" region, were presumably damaged, which further supports the proposed theory that these lesions may have caused the RBD.

This case report provides evidence that treatment aimed at the primary disease causing RBD may partially improve the frequency of RBD.

We acknowledge that some limitations are associated with this case report. Performing a follow-up PSG after the administration of chemotherapy is therefore warranted.

The authors state that they have no Conflict of Interest (COI).

\section{References}

1. American Academy of Sleep Medicine. The international classification of sleep disorders. 2nd ed. American Academy of Sleep Medicine, Westchester, IL, 2005.

2. Iranzo A, Santamaria J, Tolosa E. The clinical and pathophysiological relevance of REM sleep behavior disorder in neurodegenerative diseases. Sleep Med Rev 13: 385-401, 2009.

3. Boeve BF, Silber MH, Saper CB, et al. Pathophysiology of REM sleep behavior disorder and relevance to neurodegenerative disease. Brain 130 (Pt 11): 2770-2788, 2007.

4. Iranzo A, Ratti PL, Casanova-Molla J, Serradell M, Vilaseca I, Santamaria J. Excessive muscle activity increases over time in idiopathic REM sleep behavior disorder. Sleep 32: 1149-1153, 2009.

5. Lin FC, Liu CK, Hsu CY. Rapid-eye-movement sleep behavior disorder secondary to acute aseptic limbic encephalitis. J Neurol
256: 1174-1176, 2009.

6. Gagnon JF, Postuma RB, Mazza S, Doyon J, Montplaisir J. Rapid-eye-movement sleep behaviour disorder and neurodegenerative diseases. Lancet Neurol 5: 424-432, 2006.

7. Chokroverty S. Sleep and neurodegenerative diseases. Semin Neurol 129: 446-467, 2009.

8. Brass SD, Duquette P, Proulx-Therrien J, Auerbach S. Sleep disorders in patients with multiple sclerosis. Sleep Med Rev 14: 121$129,2010$.

9. Xi Z, Luning W. REM sleep behavior disorder in a patient with pontine stroke. Sleep Med 10: 143-146, 2009.

10. Kimura K, Tachibana N, Kohyama J, Otsuka Y, Fukazawa S, Waki $\mathrm{R}$. A discrete pontine ischemic lesion could cause REM sleep behavior disorder. Neurology 26: 894-895, 2000.

11. Plazzi G, Montagna P. Remitting REM sleep behavior disorder as the initial sign of multiple sclerosis. Sleep Med 3: 437-439, 2002.

12. Provini F, Vetrugno R, Pastorelli F, et al. Status dissociates after surgery for tegmental ponto-mesencephalic cavernoma: a statedependent disorder of motor control during sleep. Mov Disord 19: 719-723, 2004.

13. Tippmann-Peikert M, Boeve BF, Keegan BM. REM sleep behavior disorder initiated by acute brainstem multiple sclerosis. Neurology 66: 1277-1279, 2006.

14. Condurso R, Rico I, Romanello G, et al. Status dissociates in multilacunar encephalopathy with median pontine lesion: a videopolygraphic presentation. J Sleep Res 15(Suppl 1): 212, 2006.

15. Matchis J, Hess CW, Bassetti C. Isolated mediotegmental lesion causing narcolepsy and rapid eye movement sleep behaviour disorder: a case evidencing a common pathway in narcolepsy and rapid eye movement sleep behaviour disorder. J Neurol Neurosurg Psychiatry 78: 427-429, 2007.

16. Gomez-Choco MJ, Iranzo A, Blanco Y, Graus F, Santamaria J, Saiz A. Prevalence of restless legs syndrome and REM sleep behavior disorder in multiple sclerosis. Mult Scler 13: 805-808, 2007.

17. Iranzo A, Aparicio J. A lesson from anatomy: focal brain lesions causing REM sleep behavior disorder. Sleep Med 10: 9-12, 2009.

18. Limousin N, Dehais C, Gout O, et al. A brainstem inflammatory lesion causing REM sleep behavior disorder and sleepwalking (parasomnia overlap disorder). Sleep Med 10: 1059-1062, 2009.

19. Gualco G, Weiss LM, Barber GN, et al. Diffuse large B-cell lymphoma involving the central nervous system. Int J Surg Pathol 19: 44-45, 2011.

20. Sierra del Rio M, Rousseau A, Soussain C, et al. Primary CNS lymphoma in immunocompetent patients. Oncologist 14: 526-539, 2009.

21. Boeve BF. REM sleep behavior disorder: updated review of the core features, the REM sleep behavior disorder-neurodegenrative diaseases association, evolving concepts, controversies, and future directions. Ann NY Acad Sci 1184: 15-54, 2010.

22. Mahowald MW, Schenck CH. REM sleep behavior disorder. In: Principles and Practice of Sleep Medicine. 3rd ed. Kryger MH, Roth T, Dement WC, Eds. WB Saunders, Philadelphia, 2000: 724741.

23. Iranzo A, Graus F, Clover L, et al. Rapid eye movement sleep behaveior disorder and potassium channel antibody-associated limbic encephalitis. Ann Neurol 59: 178-181, 2006.

24. Lin FC, Liu CK, Hsu CY. Rapid-eye-movement sleep behavior disorder secondary to acute aseptic limbic encephalitis. J Neurol 256: 1174-1176, 2009.

25. Compta Y, Iranzo A, Santamaria J, Casamitjana R, Graus F. REM sleep behavior disorder and narcoleptic features in anti-Ma2associated encephalitis. Sleep 30: 767-769, 2007.

26. Maquet P, Peters J, Aerts J, et al. Functional neuroanatomy of human rapid-eye-movement sleep and dreaming. Nature 383: $163-$ $166,1996$. 
Intern Med 52: 617-621, 2013 DOI: 10.2169/internalmedicine.52.8786

27. Maquet P. Functional neuroimaging of normal human sleep by positron emission tomogramphy. J Sleep Res 9: 207-231, 2000.

28. Adams C, Mckeon A, Silber MH, Kumar R. Narcolepsy, REM sleep behavior disorder, and supranuclear gaze palsy associated with Ma1 and Ma2 antibodies and tonsillar carcinoma. Arch Neurol 68: $521-524,2011$

(C) 2013 The Japanese Society of Internal Medicine http://www.naika.or.jp/imonline/index.html 